\title{
Smart Demand Response Based on Smart Homes
}

\author{
Jingang Lai, Hong Zhou, Wenshan Hu, Dongguo Zhou, and Liang Zhong
}

Department of Automation, Wuhan University, Wuhan 430072, China

Correspondence should be addressed to Wenshan Hu; wenshan.hu@whu.edu.cn

Received 12 August 2014; Accepted 30 September 2014

Academic Editor: Yun-Bo Zhao

Copyright (C) 2015 Jingang Lai et al. This is an open access article distributed under the Creative Commons Attribution License, which permits unrestricted use, distribution, and reproduction in any medium, provided the original work is properly cited.

\begin{abstract}
Smart homes (SHs) are crucial parts for demand response management (DRM) of smart grid (SG). The aim of SHs based demand response (DR) is to provide a flexible two-way energy feedback whilst (or shortly after) the consumption occurs. It can potentially persuade end-users to achieve energy saving and cooperate with the electricity producer or supplier to maintain balance between the electricity supply and demand through the method of peak shaving and valley filling. However, existing solutions are challenged by the lack of consideration between the wide application of fiber power cable to the home (FPCTTH) and related users' behaviors. Based on the new network infrastructure, the design and development of smart DR systems based on SHs are related with not only functionalities as security, convenience, and comfort, but also energy savings. A new multirouting protocol based on Kruskal's algorithm is designed for the reliability and safety of the SHs distribution network. The benefits of FPCTTH-based SHs are summarized at the end of the paper.
\end{abstract}

\section{Introduction}

The whole world including American, European, Chinese, Indian, and other major economics starts to pay more attention to DRM and implement strict policies in order to achieve energy saving and $\mathrm{CO}_{2}$ reductions [1-4]. The pursuit of low-carbon economic and energy efficiency has strongly motivated the development of SG and energy management technologies $[5,6]$. DR-based energy management which is able to improve energy efficiency and manageability of current power network is a crucial research topic of SGs. On the terminal user side, DR-based energy management systems, which are usually integrated with SHs systems, play an important role in the control and optimization of domestic energy consumption and enable increasing consumer participation [7-9]. Meanwhile, the energy network on SHs has received extensive attentions due to its flexible integration into people's daily lives, which could trigger significant commercial value to develop smart applications and corresponding services for ubiquitous homes [10]. These systems offer the endusers detailed information about their energy consumption patterns, which could potentially persuade them to adopt more energy efficient behaviors.
For many years, State Grid Corporation of China has been prompting the "strong smart grid" development strategy, whose main content is to build a modern power grid with ultrahigh voltage power transmission as the backbone, and the coordinated development of power grid at all levels, such as the features of information technology, automation, and interactions. In China, FPCTTH technologies are being widely used in the constructions of SG. The adoption of optical fibers provides strong communications capacities for the power cables. Apart from transmitting the control signals for the SG control, Power cables can also provide services for internet, telephones, and television to realize the "quadruple play." This paper concentrates on the issues with respect to the design and implementation of SHs based on FPCTTH, which are the key element for the DR of SG.

The ubiquitous SHs networks have gradually evolved into complex systems to deal with various tasks, such as security, environment control, health, and also energy management. To achieve the energy saving targets, the establishment of efficient two-way information exchanges between end-users and power utility is utterly important. In the near future, $\mathrm{SH}$ s will be expanded into large networks, called smart communities or smart cities, which provide potentially larger 
scale energy savings and other relevant power management services. However, without reliable information exchange channels, the smart appliances are not able to implement effective energy saving strategies regarding the official energy policies on SG, which could negatively affect the implementation of DR policies.

As a part of SG, SHs systems with the characters and functions of DRM have gained great attention from the State Grid Corporation of China. It has funded multiple projects on large-scale domestic FPCTTH energy monitoring, response and management. With adequate information provided for the users, rational analysis and feedback based on their energy costs can be made to persuade them to change behaviors towards more environmentally friendly and energy efficient patterns.

All the discussions in this paper are based on the FPCTTH with tree topology which have more than enough bandwidth capacities to offer various services, such as domestic energy monitoring, response, and management. In Section 2, some related works, such as the role of SHs in the DR programs and the optimization of energy networks, are introduced. The system architecture and relative technical issues on its implementation are discussed in Section 3. In Section 4, the details about how SHs based on FPCTTH play a more active role to offer relevant benefits to end-users are explained before the conclusions are made in Section 5.

\section{Role of SHs in DRM}

DRM employs the flexible control in reducing the electricity consumption and peak loads to seek a balance between supply and demand of electrical energy [11]. It can achieve better utilization of the available energy and more stable and effective operation of power industry. DR involves all conscious modifications of electricity usage patterns by endusers that are intended to change electricity time, instantaneous demand level, or total electricity consumption [12]. End-users are able to actively take part in the power market management via adjusting their power consumption patterns rather than passively receiving fixed electricity prices, which gives benefits to both the power utilities and end-users.

At present, the researches mainly focus on how to put forward the concrete programs on DR from the point of view of power generation business or management [13]. They mainly address the incentive-based programs such as direct control and market based demand bidding. However, how to actually implement these programs is not their primary consideration. With the fast development of SHs and FPCTTH, end-users are able to exchange information with their power producers through the information service platform. Through the reliable communication channels provided by the fiber power cables, DR policies can be easily implemented. As shown in Figure 1, the fiber power cables, which transmit both electricity and information, are able to form a closed loop between end-users and power grid enterprise.
The conventional methods to achieve DRM are pricebased measures and incentive-based measures [10]. Pricebased measures are based on dynamic pricing rates which fluctuate following the real-time costs of electricity. The final purpose is to keep the demand curve smooth by imposing high prices during peak periods and low prices during valley periods. The basic type of price-based programs is the time of use rates, which are the rates of electricity price per unit consumption that varies in different blocks of time. The rate design attempts to reflect the average cost of electricity during different periods [14]. During contingencies, critical peak pricing rates with a prespecified even higher price are imposed to the users $[15,16]$.

Incentive-based measures are composed of direct load control measures, interruptible/curtailable load measures, emergency DR measures, and so forth. End-users who agree to cooperate with DRM are rewarded with credits according to the amount of load reduction during critical conditions. However, as the price, power utilities may seek some kinds of direct control on the users electrical consumption. For example, in case of contingencies, they may require the authorities to shut down participants' non-time-critical equipment such as air conditioners or water heaters remotely [5].

In order to implement these measures, SHs systems are becoming the bridge between DRM and SG, since SHs systems are able to simultaneously and effectively communicate between the energy services and households. To achieve domestic energy saving, energy monitoring technologies that provide real-time feedback on energy consumption (e.g., time of use or real-time pricing) are being deployed worldwide. It can potentially reduce the energy usage by up to $15 \%$ [3]. Some well-known companies, including ABB, Honeywell, and Siemens, begin to focus on the energy management for smart electricity consumption. The analysis of electric energy data [17] gives the end-users the detailed information about the consumption. However, these studies are lacking the participation from the power industries. Although the domestic Haier's U-Home solutions have been put into application in several residential real estates [18], they are trying to derive information interpretability in the device layer of customers home. However, it requires dedicated communication link and home appliances and is subject to the operators' support of communications network $[5,19]$.

Although $[20,21]$ realize the intelligent management for the refrigerator and water heater, their DR systems are isolated from the latest SG development, without building AMI mesh networks [22] and popularizing the technology to the SHs. Power line communication has been used to transmit related data for SHs [23]; however, these wired systems have limitations on scalability and resiliency and are also lacking comprehensive security notion that satisfies the needs for security-critical SHs services. On the contrary, the implementation of ZigBee-based communication provides significant advantages comparing with conventional communication technologies [24], such as rapid deployment, low cost, flexibility, and aggregated intelligence through parallel processing. In $[25,26]$, the implementation of ZigBee-based communication in the demand side of SG is introduced. 


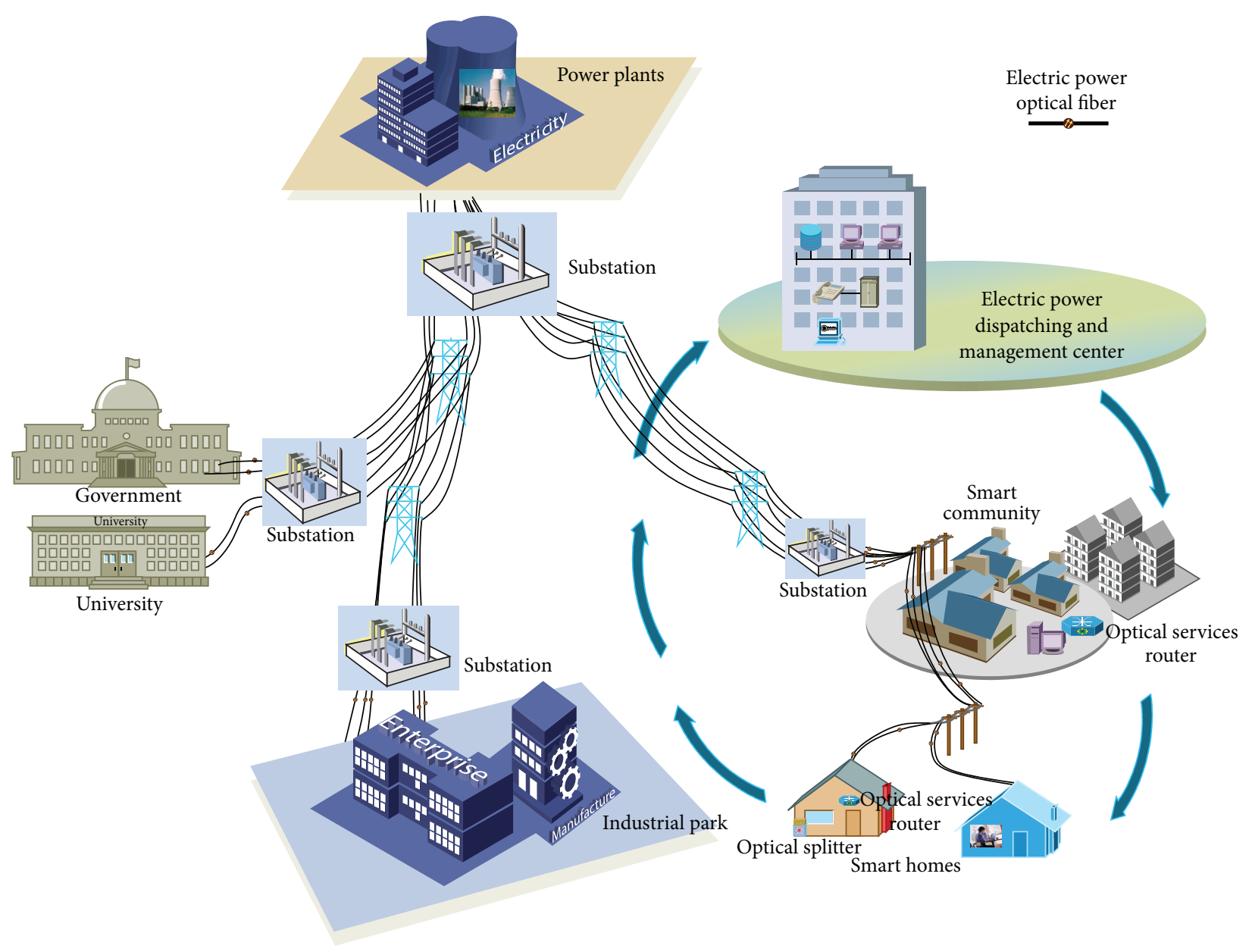

FIgURE 1: System architecture of the FPCTTH-based SG.

In $[22,27]$, SG network security issues in a wide forecasting volume and advanced measurement systems are discussed.

\section{SHs System Architecture}

SHs are able to potentially achieve energy savings and other energy-related services as long as effective two-way information exchange bridges between the world of energy service and the household world can be implemented. Due to the high communication capacity, FPCTTH can easily provide synchronous transmission of energy and massive information.

3.1. Overall Structure. The SHs network architecture proposed in the paper is as shown in Figure 2. This system is designed to coordinate and control the smart household appliances via "quadruple mode" based on FPCTTH with tree topology. The combination of distributed and centralized monitoring is carried out in the whole network architecture. In order to get the energy monitoring, demand side response, and management for SHs, the distributed monitoring mode is used not only to collect and analyze data with the help from higher layers or other distributed systems, but also to take the necessary decisions according to the calculation of local control, without any interventions from the control center. This system has a hierarchical network architecture as shown in Figure 2.

3.2. In-Home Control. The main element of in-home architectures is a ARM based smart terminal which is connected with all the indoor appliances, sensors, and actuators. A human-machine interface is provided for the users to manage all the home devices.

Figure 2 gives an overview of the proposed SHs system in the environment of smart community. The system is able to control various in-home appliances such as lamps, air conditioners, curtains, electric water heaters, and TVs. It can also be accessed using various internet terminals such as PC, tablets, and smart phones. Lots of sensors are deployed to measure the electricity consumption, indoor environment, security alarm, and so forth.

Further information about the detailed design and implementation of the FPCTTH-based SHs can be found in the early work [28]. The implementation in the laboratory environment is shown in Figure 3, where various components such as sensors and smart appliances are deployed. 


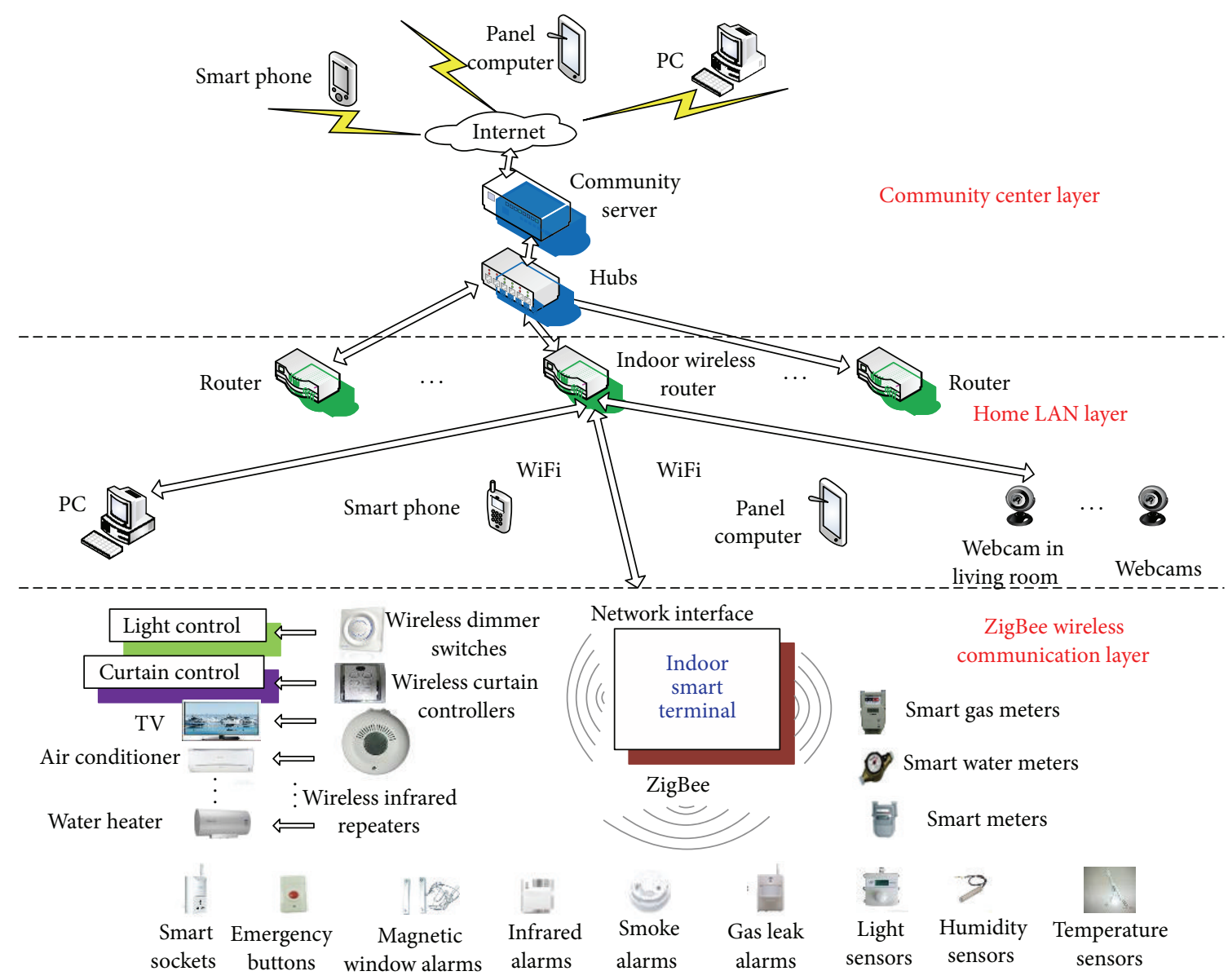

FIgURE 2: System architecture of the SHs.

They communicate with the indoor smart terminal via $2.5 \mathrm{GHz}$ ZigBee transmitter in real time. The electricity consumption data such as power consumption, transient power, electricity consumption fare during on-peak periods and off-peak periods, and maximum current/power value are collected every ten seconds. These data can be exchanged between the power utilities and end-users through the FPCTTH-based communication channels.

3.3. System Network. The solution presented in this paper selects optical fiber composite low-voltage cable and ZigBee as primary communication technologies, also proposes an IP-based communication protocol between the indoor home smart terminals and the control center.

As the fiber power cable has big communication capacity, apart from transmitting power, it can also provide services for internet, telephone, and television to form the so-called "quadruple play technology" as shown in Figure 4. Using the "quadruple play technology", the internet, telephone, and television signals are transmitted through the fiber power cable. As it provides full solutions covering both power and communication services for end-users, theoretically other cabling towards the household is no longer necessary.
The proposed system supports the access from various smart terminals, such as PC, smart phones, and tablets. In the system network, there are in-home smart sensors, actuators, and controllers which support multiple communication technologies such as Ethernet, WiFi, and ZigBee. Ethernet acts as a bridge between Internet and smart terminals,so that the system can provide HTML web page browsing services. End-users can check their current home environment using the web browser anywhere, anytime. Since the wireless AP is also connected to the system, end-users can access the home HTTP server through laptops and PADs in the house. Itinerant users can access those home services via mobile devices.

In the home LAN layer, the quadruple signals are decoded into television signals, telephone signals, and Internet signals by an optical network unit. Inside the house, smart terminals are used to manage all of the household equipment, such as security sensors, smart meters, and smart sockets. Smart terminals communicate with the smart household devices via ZigBee-based wireless communication.

The pervasive ZigBee technology is adopted for in-home transmission of sensors/actuators due to very low battery consumption and also offering specific profiles for both the home automation and energy measurement. However, it can 

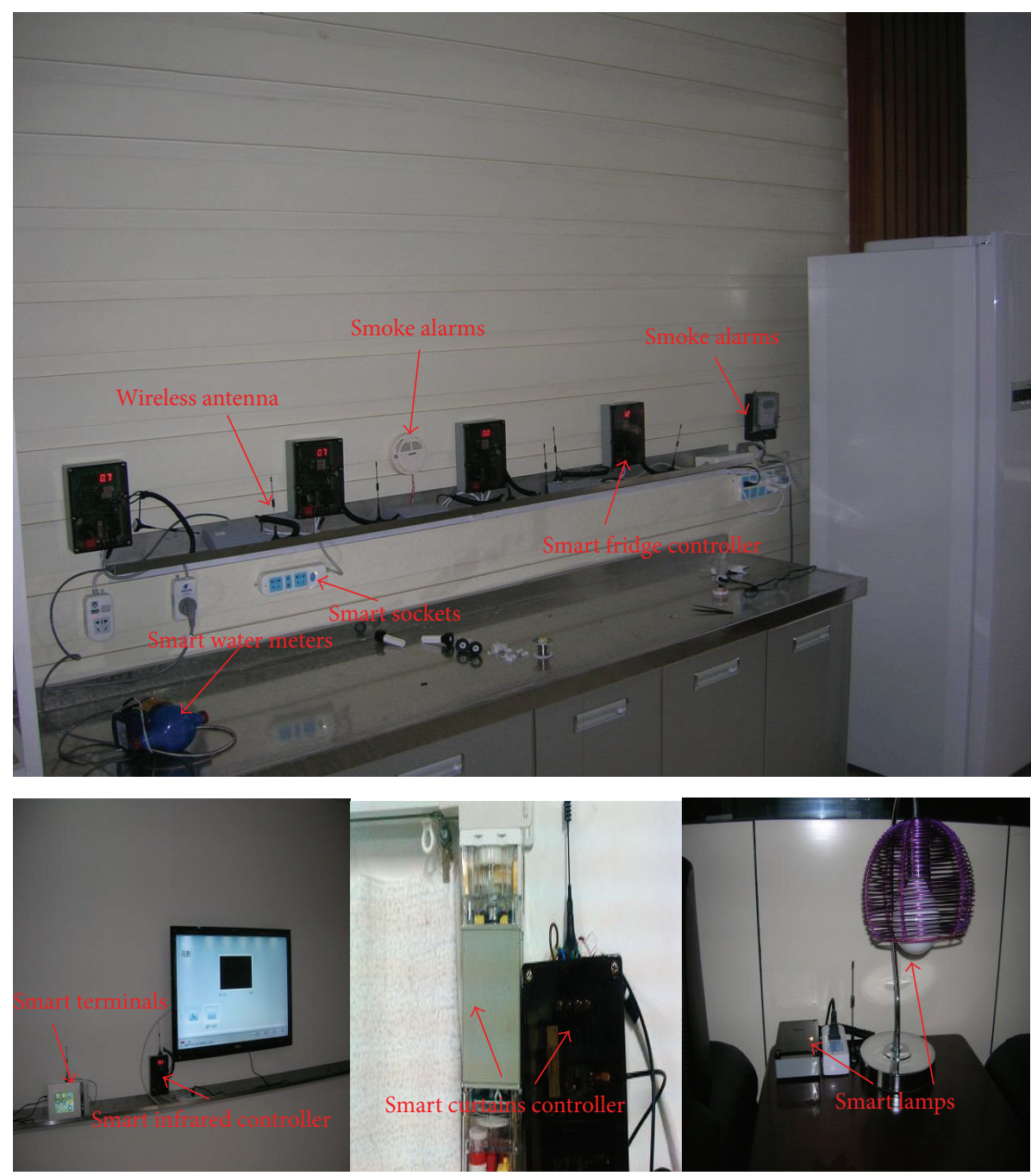

FIgURE 3: SHs indoor physical map.

be a problem with tall buildings and with thick walls for ZigBee information transmission. It can be overcome owing to the inherent structure (mesh) of the wireless standard, where intermediate sensors/actuators operate as repeaters. Thus, it is necessary to create within each building an infrastructure with a number of distributed sensors/actuators in order to ensure proper connections and full coverage.

3.4. Multipath Routing Connection Protocol. In order to ensure a proper connection and full coverage, proper routing protocols are designed to manage the distributed sensors/actuators. Each sensor/actuator has multihop routing protocol to automatically establish the wireless network between smart nodes. Then, end-users or service-providers, at the house location, can utilize the messages directly as long as the same protocol is deployed for meter data transmission and home automation services. Nevertheless, conventional routing protocols are difficult to adapt with the characters of dynamic topology variations like the proposed SHs system like the proposed SHs system. Therefore, a new routing protocol is designed based on Kruskal's algorithm named multipath routing connection protocol (MPRCP) for the wireless networks on SHs. The formation of the network is based on the MPRCP value by means of measuring from the $\mathrm{RF}$ radio.

The specific operation mechanism is as follows. The communication network of a $\mathrm{SH}$ can be modeled by a digraph. The sensors/actuators are considered as the nodes of the communication digraph. The complex weighted graph is $\mathscr{G}(\mathscr{V}, \mathscr{E}, \mathscr{A})$ with node set $\mathscr{V}=\{1,2, \ldots, n\}$, edge set $\mathscr{E}$, and symmetric edge weights $\mathscr{A}_{i j}=\mathscr{A}_{j i}$ involving link costs of time-delay, bandwidth, and traffic load for every branch $(i, j) \in \varepsilon$. The state of a node $\mathscr{V}_{i j}$ is defined as follows:

$$
\begin{aligned}
& \mathscr{V}_{i j} \\
& = \begin{cases}1, & \text { if there is the link from node } i \text { to } j \text { in solution, } \\
0, & \text { otherwise. }\end{cases}
\end{aligned}
$$




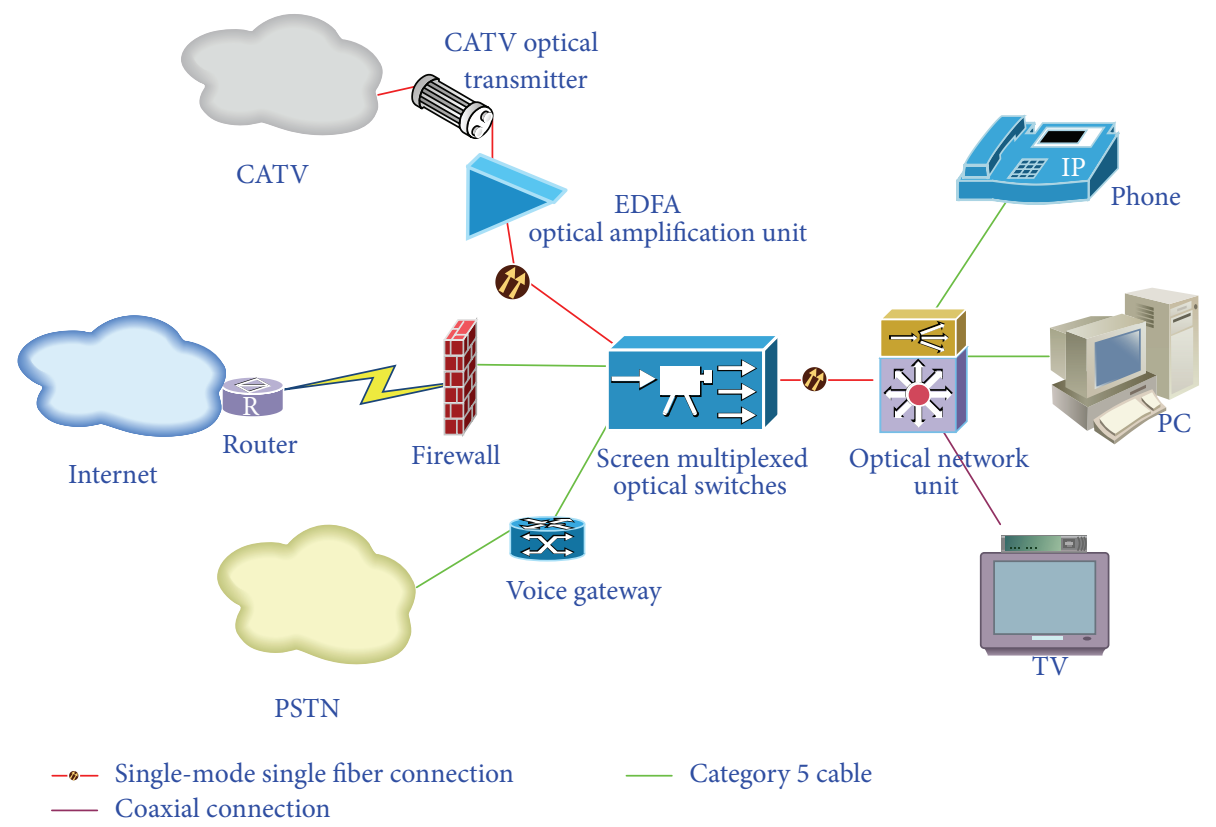

FIGURE 4: Quadruple play network structure.

A matrix $\mathscr{E}=\left[\mathscr{E}_{i j}\right]$ is defined for the link connection (or edge) as follows:

$$
\mathscr{E}_{i j}= \begin{cases}1, & \text { if there is the link from node } i \text { to } j \\ 0, & \text { otherwise. }\end{cases}
$$

If there is no link from node $i$ to $j, \mathscr{A}_{i j}$ is set to a very large value so as to reject it from the routing path. $P$ is the source node set, and $Q$ is the destination node set. For multiple destinations problem, define $q \in Q$ as the set of destinations where $q_{t} \in \mathscr{V}, t=\{1, \ldots, n\} \in \mathscr{T}$. When $P$ is nonempty and $n$ is not yet spanning, we remove a node with minimum energy from $P$. If that node connects two different networks, then add it to the network, combining two networks into one network; otherwise, reject that node.

If an undirected routing regarding multiple destinations $q$ is defined as follows [29]:

$$
\operatorname{Path}^{p q_{t}}=\left(p, \alpha, \beta, \ldots, q_{1}, \ldots, q_{2}, \ldots, i, q_{t}\right)
$$

that is to say, the routing is $\left(p \rightarrow \alpha \rightarrow \beta \rightarrow \cdots \rightarrow q_{1} \rightarrow\right.$ $\cdots \rightarrow q_{2} \rightarrow \cdots \rightarrow i \rightarrow q_{t}$ ), then the total cost [TotalCost] $p q_{t}$ of this issue is turned into [TotalCost] ${ }_{p q_{t}}=$ $\mathscr{A}_{p \alpha} \mathscr{E}_{p \alpha}+\mathscr{A}_{\alpha \beta} \mathscr{E}_{\alpha \beta}+\cdots+\mathscr{A}_{i p_{t}} \mathscr{E}_{i p_{t}}$.

According to the above definitions, the problem of multiple destinations can describe the constrained combinatorial optimization issue; the expressions are as follows:

$$
\operatorname{Minimize} \sum_{i=0}^{n-1} \sum_{j=0}^{n-1} \mathscr{A}_{i j} \mathscr{E}_{i j}
$$

for all $\left(p \rightarrow q_{t}\right)$

$$
\text { Subject to }\left(\sum_{\substack{j=0 \\ j \neq i}}^{n-1} \mathscr{A}_{i j}-\sum_{\substack{k=0 \\ k \neq i}}^{n-1} \mathscr{A}_{k i}\right)= \begin{cases}1, & \text { if } i=p, \\ -1, & \text { if } i=q_{t}, \\ 0, & \text { otherwise. }\end{cases}
$$

At the termination of the algorithm, the network has only one component and forms a minimum spanning network of the graph. It is shown that Kruskal's algorithm is run $0(n \log n)$ times or equivalently $0(n \log n)$ time, all with simple data structures. These running times are equivalent [25].

With the proposed tree topology MPRCP, each node only needs to communicate with its neighboring nodes intermittently to select the special node having the best MPRCP value even if their communication networks are local, while the data packet is put forward to the sink node. Meanwhile, the topology of routing path is dynamically adjusted because every node continuously monitors the neighbor information on the lists of MPRCP whenever exchanging information. The MAC protocol for shared media access is introduced, and the MPRCP value is provided by a special RF device with narrow band.

3.5. Home Security. In this section, the security issues of ZigBee-based wireless communication scheme for SHs are studied. All the data collected from the smart homes are related to the users daily lives. They could be of great privacy and should not be tapped during the communication. The completeness and precision of sensitive information must be protected against possible malicious attacks.

In the proposed SHs system, encryption security gateways are set up in the community layer to keep the sensitive 


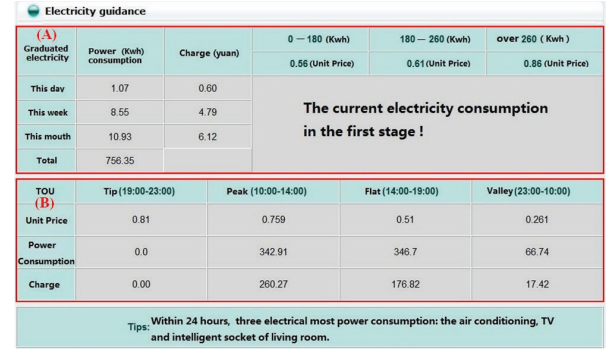

FIGURE 5: Snapshots of the dashboard pages on incentive-based demand response. Features are highlighted by red rectangles, graduate price on current energy consumption of each appliance using web technologies; (B) time of use (TOU) on current energy consumption of each appliance using web technologies.

information secure. The whole data encryption process for in-home security is divided into two stages: firstly, each sensor/actuator must be configured at the time of installation. Using this initial knowledge, each sensor/actuator is able to prove its identity and may dynamically join one or more secure communication relationships at the time of first running. Secondly, within such a secure relationship, the sensor/actuator is able to exchange data through a secured channel. Symmetric cryptographic algorithms are used to realize the objectives of a secured channel. A typical example could be the use of encryption algorithms like DES to guarantee data confidentiality and a MAC algorithm to guarantee data integrity.

\section{Benefits of Smart Home}

The SHs implement brings significant benefits to the electricity suppliers within a medium or short time. It supplies a more prompt and accurate billing information for the end-users and helps them to reduce the energy costs by adopting more energy efficient and environmentally friendly life styles. Nonetheless, a complete exploitation of DR in SG advantages can only be achieved when efficient FPCTTHbased communication channels between the energy services department and the household are established. The endusers also can enjoy lots of benefits as they have detailed information and more control about their electricity usage. Consider the following.

(1) From the aspect of consumption information and energy saving awareness for end-users, the proposed system lets end-users know their detailed consumption. Therefore, they are encouraged to change their behaviors to reduce the energy costs. Through the web-based interface as shown in Figures 5 and 6, endusers are able to intuitively see all kinds of home appliances, power consumption information, and the corresponding costs clearly.

(2) From the aspect of interactive services for DRM, the purpose of incentive DR policies is to make full use of mechanisms of the energy market and the differentiation on energy rate during the day to save

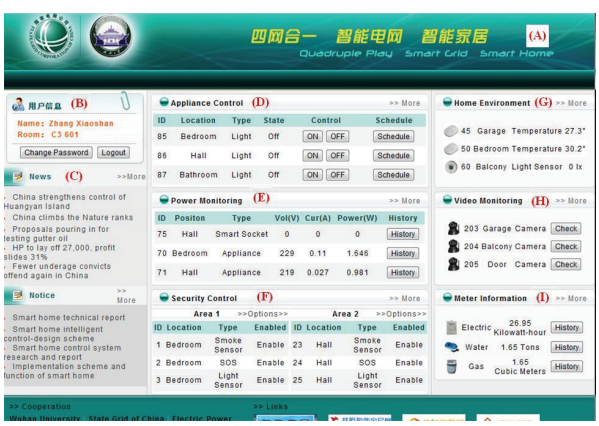

FIGURE 6: Snapshots of electricity guidance using the third-party web or app technologies. Features are highlighted by red rectangles, including (A) navigation panel, (B) user info and network status, (C) news, (D) appliance control, (E) energy savings compared to historical usage and live energy usage, (F) monitoring alarms, $(\mathrm{G})$ indoor environment, $(\mathrm{H})$ video surveillance, and (I) three-meter information and history curve of current energy usage using web technologies.

the consumption costs for end-users. End-users are able to take these actions, such as the reduction of the power supply based on the information from the management platform (as shown in Figure 5) and the choice of usage hours at low price, to achieve energy saving. When end-users can easily see their consumption information, they would voluntarily perform relevant policies. Figure 6 shows incentivebased DR options including remote control of customer's electrical equipment for a short notice or the end-users reducing load during system contingencies in return for rate discounts.

\section{Conclusion}

In this paper, a novel DRM based on SHs and FPCTTH system has been introduced. The use of FPCTTH provides reliable communication channels for the interaction between electricity suppliers and end-users. The advantage of FPCTTH is that there is a considerable reduction of cabling and it can be achieved through a simple extension for the low-voltage lines of the previously installed network. The SHs system proposed in this paper has been implemented in the laboratories of State Grid Technology College in China. It is able to create an interactive environment for end-users to actually understand how the electricity is consumed and what is the real-time costs. With all the information which can be accessed through a web-based interface, the users can be encouraged to adjust their behaviors and consume less energy. ZigBee technologies are also used for the communications between the indoor smart terminal and various household smart appliances. In order to keep the radiabilities of the ZigBee communication for the SHs system, a new MPRCP based on Kruskal's algorithm is introduced in the paper. 


\section{Conflict of Interests}

The authors declare that there is no conflict of interests regarding the publication of this paper.

\section{Acknowledgments}

This work was supported by National Key Technology Support Program of China under Grant 2013BAA01B01, by the National Natural Science Foundation of China under Grant 61374064, and by the Fundamental Research Funds for the Central Universities under Grant 2014208020201.

\section{References}

[1] S. M. Amin and B. F. Wollenberg, "Toward a smart grid: power delivery for the 21st century," IEEE Power and Energy Magazine, vol. 3, no. 5, pp. 34-41, 2005.

[2] H. Farhangi, "The path of the smart grid," IEEE Power and Energy Magazine, vol. 2, pp. 18-28, 2010.

[3] C.-C. Lin, C.-H. Yang, and J. Z. Shyua, "A comparison of innovation policy in the smart grid industry across the pacific: China and the USA," Energy Policy, vol. 57, pp. 119-132, 2013.

[4] Q. Liu, G. Cooper, N. Linge, H. Takruri, and R. Sowden, "DEHEMS: creating a digital environment for large-scale energy management at homes," IEEE Transactions on Consumer Electronics, vol. 59, no. 1, pp. 62-69, 2013.

[5] P. Palensky and D. Dietrich, "Demand side management: demand response, intelligent energy systems, and smart loads," IEEE Transactions on Industrial Informatics, vol. 7, no. 3, pp. 381388, 2011.

[6] D. Dietrich, D. Bruckner, G. Zucker, and P. Palensky, "Communication and computation in buildings: a short introduction and overview," IEEE Transactions on Industrial Electronics, vol. 57, no. 11, pp. 3577-3584, 2010.

[7] F. Rahimi and A. Ipakchi, "Demand response as a market resource under the smart grid paradigm," IEEE Transactions on Smart Grid, vol. 1, no. 1, pp. 82-88, 2010.

[8] H. A. Aalami, M. P. Moghaddam, and G. R. Yousefi, "Demand response modeling considering interruptible/curtailable loads and capacity market programs," Applied Energy, vol. 87, no. 1, pp. 243-250, 2010.

[9] G. Strbac, "Demand side management: benefits and challenges," Energy Policy, vol. 36, no. 12, pp. 4419-4426, 2008.

[10] M. H. Albadi and E. F. El-Saadany, "A summary of demand response in electricity markets," Electric Power Systems Research, vol. 78, no. 11, pp. 1989-1996, 2008.

[11] US Department of Energy, "Benefits of demand response in electricity narkets and recommendations for achieving them," Report to the United States Congress, 2006, http://eetd.lbl.gov/.

[12] O. Sezgen, C. A. Goldman, and P. Krishnarao, "Option value of electricity demand response," Energy, vol. 32, no. 2, pp. 108-119, 2007.

[13] P. Jazayeri, A. Schellenberg, W. D. Rosehart et al., "A survey of load control programs for price and system stability," IEEE Transactions on Power Systems, vol. 20, no. 3, pp. 1504-1509, 2005.

[14] D. S. Kirschen, "Demand-side view of electricity markets," IEEE Transactions on Power Systems, vol. 18, no. 2, pp. 520-527, 2003.
[15] I. C. Paschalidis, B. Li, and M. C. Caramanis, "Demand-side management for regulation service provisioning through internal pricing," IEEE Transactions on Power Systems, vol. 27, no. 3, pp. 1531-1539, 2012.

[16] C.-L. Su and D. Kirschen, "Quantifying the effect of demand response on electricity markets," IEEE Transactions on Power Systems, vol. 24, no. 3, pp. 1199-1207, 2009.

[17] V. C. Gungor, D. Sahin, T. Kocak et al., "A Survey on smart grid potential applications and communication requirements," IEEE Transactions on Industrial Informatics, vol. 9, no. 1, pp. 28-42, 2013.

[18] L. J. Zhong, H. R. Su, and C. W. Feng, "Haier U-Home digital home system," Digital Community and Smart Home, vol. 7, pp. 21-24, 2007.

[19] M. A. Alahmad, P. G. Wheeler, A. Schwer, J. Eiden, and A. Brumbaugh, "A comparative study of three feedback devices for residential real-time energy monitoring," IEEE Transactions on Industrial Electronics, vol. 59, no. 4, pp. 2002-2013, 2012.

[20] A. Arteconi, N. J. Hewitt, and F. Polonara, "Domestic demandside management (DSM): role of heat pumps and thermal energy storage (TES) systems," Applied Thermal Engineering, vol. 51, no. 1-2, pp. 155-165, 2013.

[21] M. A. Zehir and M. Bagriyanik, "Demand side management by controlling refrigerators and its effects on consumers," Energy Conversion and Management, vol. 64, pp. 238-244, 2012.

[22] H. Li, S. Gong, L. Lai, Z. Han, R. C. Qiu, and D. Yang, "Efficient and secure wireless communications for advanced metering infrastructure in smart grids," IEEE Transactions on Smart Grid, vol. 3, no. 3, pp. 1540-1551, 2012.

[23] J. Kim, D. Kim, K.-W. Lim, Y.-B. Ko, and S.-Y. Lee, "Improving the reliability of IEEE 802.11s Based wireless mesh networks for smart grid systems," Journal of Communications and Networks, vol. 14, no. 6, pp. 629-639, 2012.

[24] F. Benzi, N. Anglani, E. Bassi, and L. Frosini, "Electricity smart meters interfacing the households," IEEE Transactions on Industrial Electronics, vol. 58, no. 10, pp. 4487-4494, 2011.

[25] D.-M. Han and J.-H. Lim, "Design and implementation of smart home energy management systems based on ZigBee," IEEE Transactions on Consumer Electronics, vol. 56, no. 3, pp. 14171425,2010

[26] V. C. Gungor, B. Lu, and G. P. Hancke, "Opportunities and challenges of wireless sensor networks in smart grid," IEEE Transactions on Industrial Electronics, vol. 57, no. 10, pp. 3557-3564, 2010.

[27] W. Granzer, F. Praus, and W. Kastner, "Security in building automation systems," IEEE Transactions on Industrial Electronics, vol. 57, no. 11, pp. 3622-3630, 2010.

[28] H. Zhou, J. G. Lai, W. Hu, and Q. Deng, "Demand-side energy management: FTTH-based mode for smart homes," in Proceedings of the American Control Conference (ACC '14), vol. 48, pp. 1704-1709, Portland, Ore, USA, June 2014.

[29] H. Chen and B. L. Sun, "Multicast routing optimization algorithm with bandwidth and delay constraints based on GA," Journal of Communication and Computer, vol. 2, no. 5, pp. 6367, 2005 . 


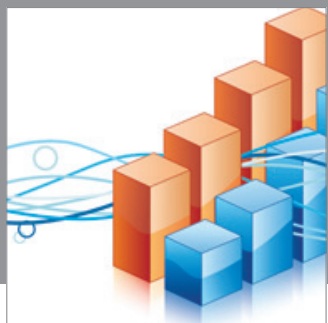

Advances in

Operations Research

mansans

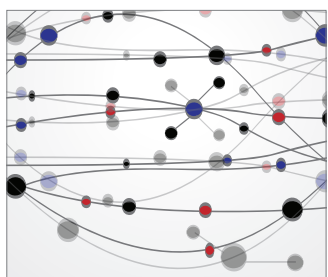

The Scientific World Journal
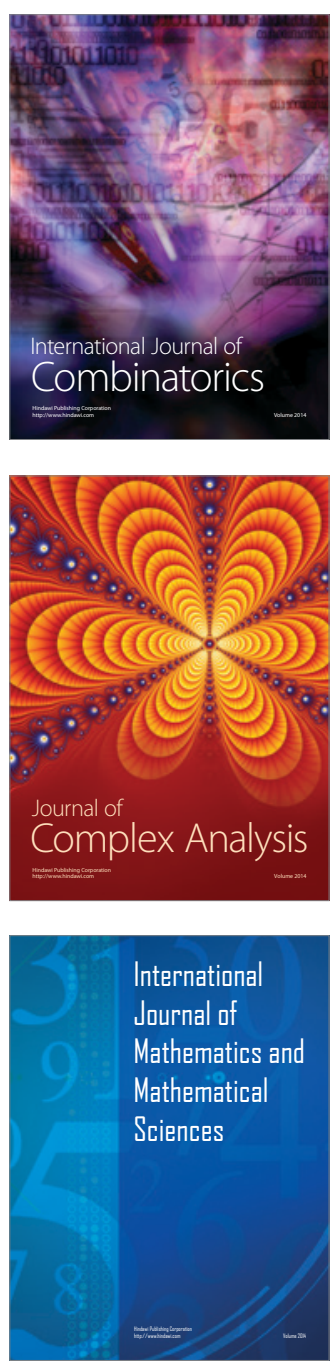
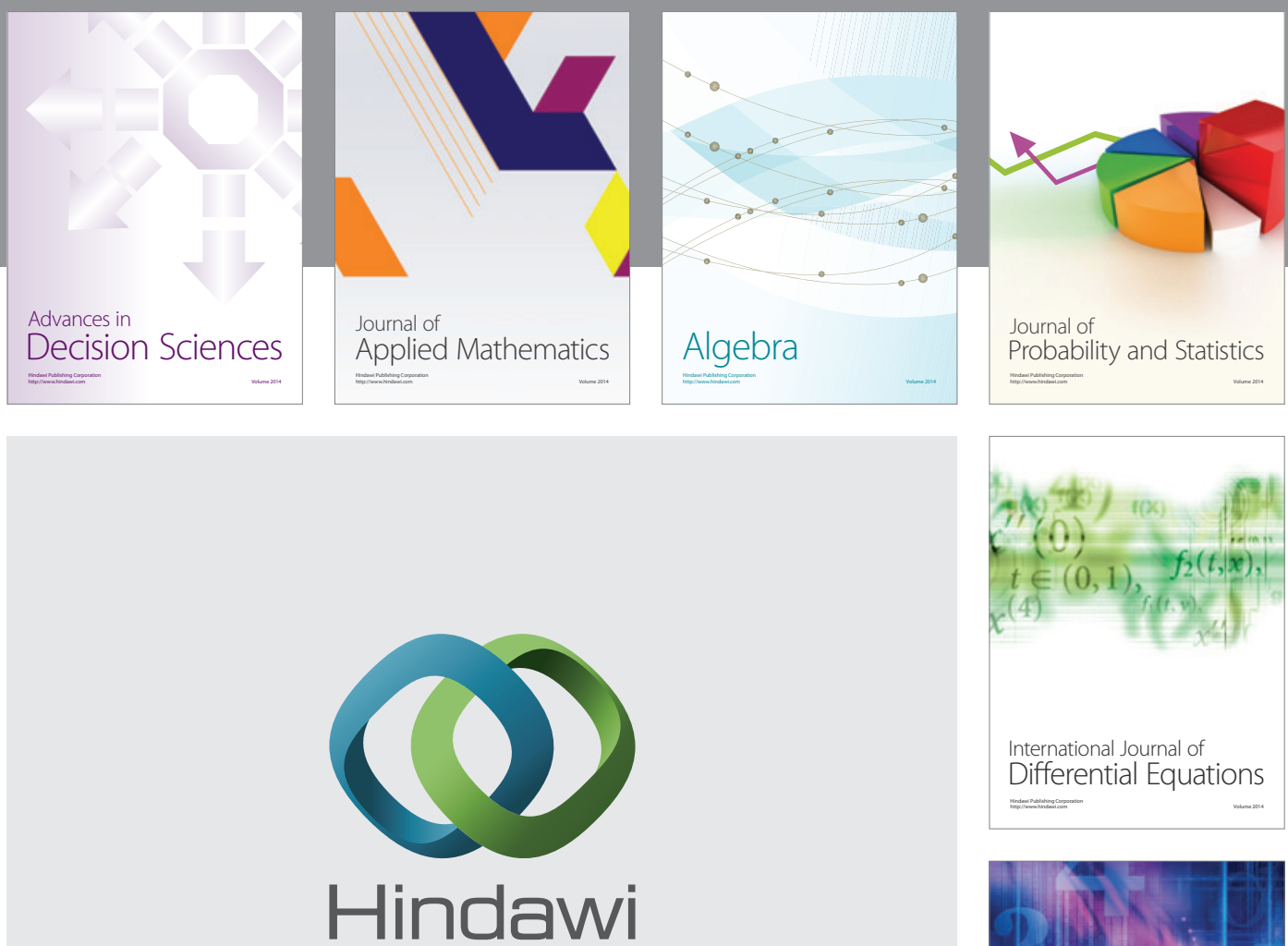

Submit your manuscripts at http://www.hindawi.com
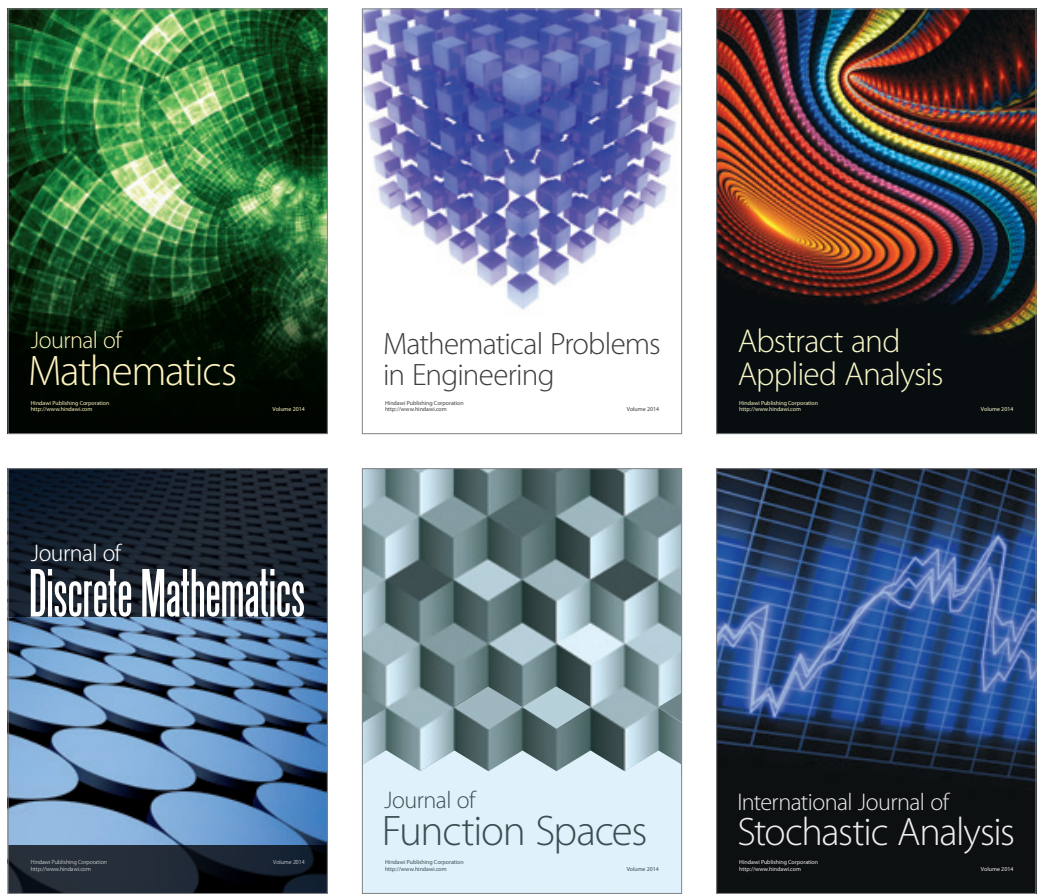

Journal of

Function Spaces

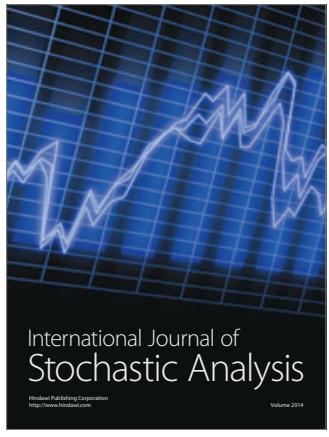

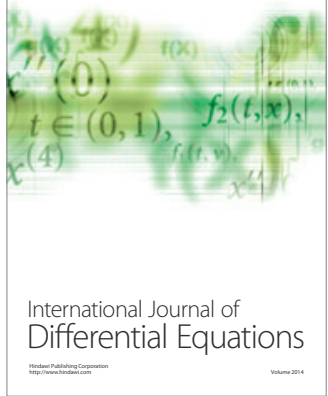
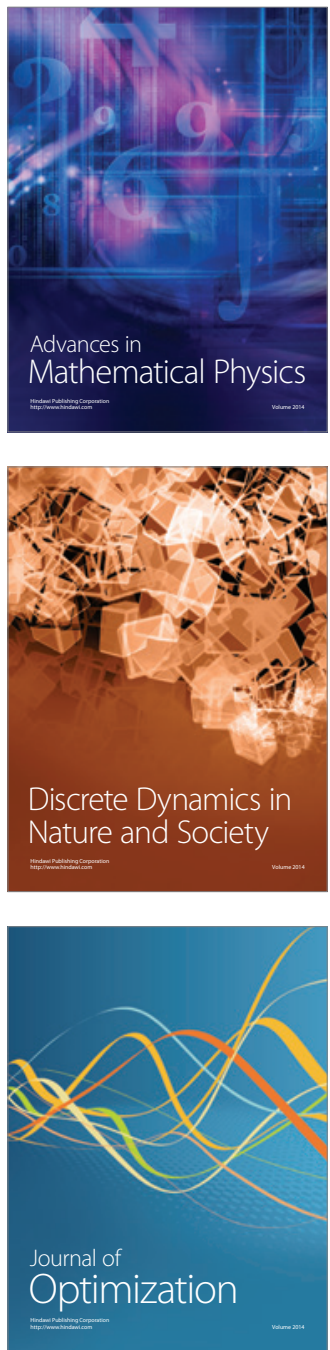